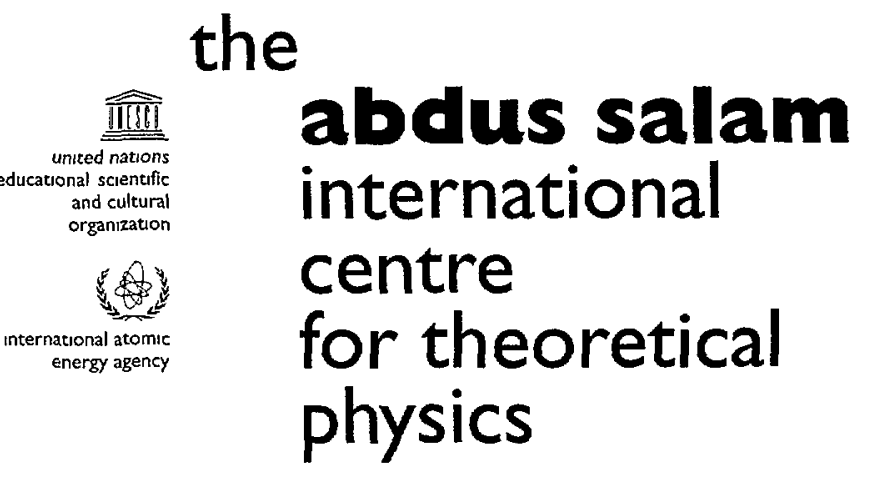

CONVERGENCE THEOREMS FOR MAPPINGS WHICH ARE ASYMPTOTICALLY NONEXPANSIVE IN THE INTERMEDIATE SENSE

\author{
C.E. Chidume
}

Naseer Shahzad

and

Habtu Zegeye
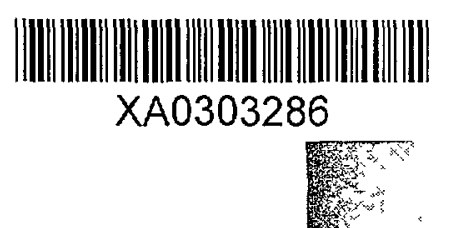
United Nations Educational Scientific and Cultural Organization

and

International Atomic Energy Agency

THE ABDUS SALAM INTERNATIONAL CENTRE FOR THEORETICAL PHYSICS

\title{
CONVERGENCE THEOREMS FOR MAPPINGS WHICH ARE ASYMPTOTICALLY NONEXPANSIVE IN THE INTERMEDIATE SENSE
}

\author{
C. E. Chidume ${ }^{1}$ \\ The Abdus Salam International Centre for Theoretical Physics, Trieste, Italy, \\ Naseer Shahzad ${ }^{2}$ \\ Department of Mathematics, King Abdul Aziz Unvversity, \\ P.O.B. 80203, Jeddah 21589, Saudi Arabia \\ and \\ The Abdus Salam International Centre for Theoretical Physics, Trieste, Italy \\ and \\ Habtu Zegeye ${ }^{3}$ \\ The Abdus Salam International Centre for Theoretical Physics, Trieste, Italy.
}

\begin{abstract}
Suppose $K$ is a nonempty closed convex nonexpansive retract of a real uniformly convex Banach space $E$ with $P$ as a nonexpansive retraction. Let $T: K \rightarrow E$ be a non-self mapping which is asymptotically nonexpansive in the intermediate sense with $F(T):=\{x \in K: T x=x\} \neq \emptyset$. A demiclosed principle for $T$ is proved. Moreover, if $T$ is completely continuous, an iterative sequence $\left\{x_{n}\right\}$ is constructed which converges strongly to some $x^{*} \in F(T)$. If $T$ is not assumed to be completely continuous but the dual $E^{*}$ of $E$ is assumed to have the Kadec-Klee property, then $\left\{x_{n}\right\}$ converges weakly to some $x^{*} \in F(T)$. The operator $P$ which plays a central role in our proofs is, in this case, the Banach space analogue of the proximity map in Hilbert spaces.
\end{abstract}

MIRAMARE - TRIESTE

August 2003

\footnotetext{
${ }^{1}$ chidume@ictp trieste.it

${ }^{2}$ Junior Associate of the Abdus Salam ICTP.naseer_shahzad@hotmail com

3habz@ictp.trieste it
} 


\section{INTRODUCTION}

Let $K$ be a nonempty subset of a real normed linear space $E$. Let $T$ be a self-mapping of $K$. Then $T$ is said to be asymptotically nonexpansive if there exists a sequence $\left\{k_{n}\right\} \subset[1, \infty)$ with $k_{n} \rightarrow 1$ as $n \rightarrow \infty$ such that $\forall x, y \in K$, the following inequality holds:

$$
\left\|T^{n} x-T^{n} y\right\| \leq k_{n}\|x-y\| \forall n \geq 1 \text {. }
$$

$T$ is called uniformly $L$-Lıpschitzian if there exists a constant $L>0$ such that $\forall x, y \in K$,

$$
\left\|T^{n} x-T^{n} y\right\| \leq L\|x-y\| \forall n \geq 1
$$

The class of asymptotically nonexpansive maps was introduced by Goebel and Kirk [12] as an important generalization of the class of nonexpansive maps (i.e., mappings $T: K \rightarrow K$ such that $\|T x-T y\| \leq$ $\|x-y\| \quad \forall x, y \in K)$.

Bruck et. al [3] introduced the notion of mappings which are asymptotically nonexpansive in the intermediate sense. The map $T$ is called asymptotically nonexpansive on the intermediate sense if $T$ is uniformly continuous and the following inequality holds:

$$
\limsup _{n \rightarrow \infty} \sup _{x, y \in K}\left(\left\|T^{n} x-T^{n} y\right\|-\|x-y\|\right) \leq 0 .
$$

It is known [19] that if $K$ is a nonempty closed convex bounded subset of a uniformly convex Banach space $E$ and $T$ is a self-map of $K$ which is asymptotically nonexpansive in the intermediate sense, then $T$ has a fixed point. It is worth mentioning that the class of mappings which are asymptotically nonexpansive in the intermediate sense contains properly the class of asymptotically nonexpansive maps.

Iterative techniques for approximating fixed points of nonexpansive mappings and asymptotically nonexpansive mappings have been studied by various authors (see e.g., [4] - [5], [8], [14], [23] - [33]), using the Mann iteration method (see e.g., [21]) or the Ishıkawa iteration method (see e.g., [15]).

In 1978, Bose [1] proved that if $K$ is a nonempty closed convex bounded subset of a uniformly convex Banach space $E$ satisfying Opial's condition and $T: K \rightarrow K$ is an asymptotically nonexpansive mapping, then the sequence $\left\{T^{n} x\right\}$ converges weakly to a fixed point of $T$ provided $T$ is asymptotically regular at $x \in K$, i.e. $\lim _{n \rightarrow \infty}\left\|T^{n} x-T^{n+1} x\right\|=0$. Passty [24] and also $\mathrm{Xu}$ [36] proved that the requirement that $E$ satisfies Opial's condition can be replaced with the condition that $E$ has a Fréchet differentiable norm. Furthermore, Tan and $\mathrm{Xu}([30,31])$ proved that the asymptotic regularity of $T$ at $x$ can be weakened to the so-called weakly asymptotic regularity of $T$ at $x$, i.e., $\omega-\lim _{n \rightarrow \infty}\left(T^{n} x-T^{n+1} x\right)=0$.

In $[27,28]$, Schu introduced a modified Mann process to approximate fixed points of asymptotically nonexpansive self-maps defined on nonempty closed convex and bounded subsets of a uniformly convex Banach space $E$. In particular, he proved the following theorem: 
Theorem JS. ([28, Theorem 2.1, p. 156]) Let E be a uniformly convex Banach space satisfying Opial's conditon and $K$ a nonempty closed convex bounded subset of $E$. Let $T: K \rightarrow K$ be an asymptotzcally nonexpansive mapping with sequence $\left\{k_{n}\right\}_{n \geq 1} \subset[1, \infty)$ for all $n \geq 1, \lim k_{n}=1$, and $\sum_{n=1}^{\infty}\left(k_{n}^{2}-1\right)<\infty$. Let $\left\{\alpha_{n}\right\}_{n \geq 1}$ be a real sequence in $(0,1)$ satisfynng the condition $0<a \leq \alpha_{n} \leq b<1, n \geq 1$, for some constants $a, b$. Then the sequence $\left\{x_{n}\right\}$ generated from arbıtrary $x_{1} \in K$ by

$$
x_{n+1}=\left(1-\alpha_{n}\right) x_{n}+\alpha_{n} T^{n} x_{n}, \quad n \geq 1
$$

converges weakly to some fixed point of $T$.

Schu's result does not apply to $L^{p}$ spaces with $p \neq 2$ because none of these spaces satisfy Opial's condition. In [26], Rhoades obtained strong convergence theorem for asymptotically nonexpansive mappings in uniformly convex Banach spaces using a modified Ishikawa iteration method. In [23], Osilike and Aniagbosor proved that the theorems of Schu and Rhoades remain true without the boundedness condition imposed on $K$, provided that $F(T)=\{x \in K: T x=x\} \neq \emptyset$. In [33], Tan and Xu extended Theorem JS to the so-called Ishikawa iteration scheme. They also obtained the following result which applies to $L^{p}$ spaces with $1<p<\infty$ since each of these spaces is uniformly convex and has a Fréchet differentiable norm.

Theorem TX. ([33, Theorem 3.1, p. 737]) Let E be a unzformly convex Banach space with a Fréchet differentiable norm and $K$ a nonempty closed convex bounded subset of $E$. Let $T: K \rightarrow K$ be an asymptotically nonexpansive mapping with sequence $\left\{k_{n}\right\}_{n \geq 1} \subset[1, \infty)$ for all $n \geq 1, \lim k_{n}=1$, and $\sum_{n=1}^{\infty}\left(k_{n}-1\right)<\infty$. Let $\left\{\alpha_{n}\right\}_{n \geq 1}$ be a real sequence in $(0,1)$ satrsfying the condition $0<a \leq \alpha_{n} \leq b<1$, $n \geq 1$, for some constants $a, b$. Then the sequence $\left\{x_{n}\right\}$ generated from arbitrary $x_{1} \in K$ by

$$
x_{n+1}=\left(1-\alpha_{n}\right) x_{n}+\alpha_{n} T^{n} x_{n}, \quad n \geq 1
$$

converges weakly to some fixed point of $T$.

Chang et al. [4] derived convergence theorems for asymptotically nonexpansive mappings and nonexpansive mappings in Banach spaces without assuming any of the following conditions: (a) $E$ satisfies Opial's condition; (b) $T$ is asymptotically regular or weakly asymptotically regular; (c) $K$ is bounded. Their results improve and generalize the corresponding results of Bose [1], Górnicki [13], Passty [24], Reich [25], Schu [28], Tan and Xu ([30,31, 33]), and Xu [36].

Recently, Kim and Kim et al. [18] studied the strong convergence of the Mann and Ishikawa iterations with errors for asymptotically nonexpansive self-mappings in the intermediate sense in Banach spaces.

In all the above results, the operator $T$ remains a self- mapping of a nonempty closed convex subset $K$ of a uniformly convex Banach space. If, however, the domain of $T, D(T)$, is a proper subset of $E$ (and this is the case in several applications), and $T$ maps $D(T)$ into $E$ then, the iteration processes of Mann and 
Ishikawa studied by these authors and their modifications introduced by Schu, may fail to be well-defined.

More recently, Chidume et al. [7] obtained convergence theorems for asymptotically nonexpansive non-self mappings in Banach spaces which extend the corresponding results of Chang et al. [4], Osilike and Aniagbosor [23] and Rhoades [26].

It is our purpose in this paper to first introduce the class of non-self mappings which are asymptotically nonexpansive in the intermediate sense and to prove demiclosed principle for such mappings. Then, we construct an iteration scheme for approximating a fixed point of any map belonging to this class (when such a fixed point exists). We prove strong and weak convergence theorems. Our theorems improve and generalize important known results (see our Remark 3.18).

\section{Preliminaries}

Let $E$ be a real normed linear space. The modulus of convexity of $E$ is the function $\delta_{E}:(0,2] \rightarrow[0,1]$ defined by

$$
\delta_{E}(\epsilon):=\inf \left\{1-\left\|\frac{x+y}{2}\right\|:\|x\|=\|y\|=1, \epsilon=\|x-y\|\right\} .
$$

$\mathrm{E}$ is uniformly convex if and only if $\delta_{E}(\epsilon)>0 \forall \epsilon \in(0,2]$.

It is well known (see e.g., $[11,20,35]$ ) that in a uniformly convex space, $\delta_{E}$ is continuous, strictly increasing, and $\delta_{E}(0)=0$.

A subset $K$ of $E$ is said to be a retract of $E$ if there exists a continuous map $P: E \rightarrow K$ such that $P x=x \forall x \in K$. Every closed convex subset of a uniformly convex Banach space is a retract. A map $P: E \rightarrow E$ is said to be a retraction if $P^{2}=P$. It follows that if a map $P$ is a retraction, then $P y=y$ for all $y$ in the range of $P$.

A mapping $T$ with domain $D(T)$ and range $R(T)$ in $E$ is said to be demiclosed at $p$ if whenever $\left\{x_{n}\right\}$ is a sequence in $D(T)$ such that $\left\{x_{n}\right\}$ converges weakly to $x^{*} \in D(T)$ and $\left\{T x_{n}\right\}$ converges strongly to $p$, then $T x^{*}=p$.

A Banach space $E$ is said to have the Kadec-Klee property if for every sequence $\left\{x_{n}\right\}$ in $E, x_{n} \rightarrow x$ weakly and $\left\|x_{n}\right\| \rightarrow\|x\|$ strongly together imply $\left\|x_{n}-x\right\| \rightarrow 0$.

In what follows, we shall make use of the following lemma.

Lemma 2.1. (see e.g., [16]) Let $E$ be a real reflexive Banach space such that its dual $E^{*}$ has the KadecKlee property. Let $\left\{x_{n}\right\}$ be a bounded sequence in $E$ and $x^{*}, y^{*} \in \omega_{w}\left(x_{n}\right)$, where $\omega_{w}\left(x_{n}\right)$ denotes the weak $w$-limit set of $\left\{x_{n}\right\}$. Suppose $\lim _{n \rightarrow \infty}\left\|t x_{n}+(1-t) x^{*}-y^{*}\right\|$ exists for all $t \in[0,1]$. Then $x^{*}=y^{*}$. 


\section{MAin RESUlts}

In this section, we give definitons and prove our main theorems.

Definition 3.1. Let $E$ be a real normed linear space and $K$ a nonempty subset of $E$. Let $P: E \rightarrow K$ be a nonexpansive retraction of $E$ onto $K$. A map $T: K \rightarrow E$ is said to be asymptotically nonexpansive if there exists a sequence $\left\{k_{n}\right\} \subset[1, \infty), k_{n} \rightarrow 1$ as $n \rightarrow \infty$ such that the following inequality holds:

$$
\left\|T(P T)^{n-1} x-T(P T)^{n-1} y\right\| \leq k_{n}\|x-y\| \quad \forall x, y \in K, n \geq 1 .
$$

$T$ is called uniformly $L$-Lipschatzian if there exists $L>0$ such that

$$
\left\|T(P T)^{n-1} x-T(P T)^{n-1} y\right\| \leq L\|x-y\| \quad \forall x, y \in K, n \geq 1 .
$$

Definition 3.2. Let $E$ be a real normed linear space and $K$ a nonempty subset of $E$. Let $P: E \rightarrow K$ be a nonexpansive retraction of $E$ onto $K$. A map $T: K \rightarrow E$ is said to be asymptotically nonexpansive in the intermediate sense if $T$ is uniformly continuous and the following inequality holds:

$$
\limsup _{n \rightarrow \infty} \sup _{x, y \in K}\left(\left\|T(P T)^{n-1} x-T(P T)^{n-1} y\right\|-\|x-y\|\right) \leq 0 \text {. }
$$

Let $\mathrm{K}$ be a nonempty closed convex subset of a real uniformly convex Banach space $\mathrm{E}$. The following iteration scheme is studied:

$$
x_{1} \in K, \quad x_{n+1}=P\left(\left(1-\alpha_{n}\right) x_{n}+\alpha_{n} T(P T)^{n-1} x_{n}\right),
$$

where $\left\{\alpha_{n}\right\}_{n \geq 1}$ is a sequence in $(0,1)$, and $P$ is as in definition 3.1 .

Remark 3.3. If $T$ is a self-map, then $P$ becomes the identity map so that (3.1), (3.2) and (3.3) coincide with (1.1), (1.2) and (1.3), respectively. Moreover, (3.4) reduces to the recursion formula (1.4) (see also formulas (1.5) and (1.6)).

In the sequel, we shall need the following lemma:

Lemma 3.4. Let $E$ be a real uniformly convex Banach space and $C$ a nonempty closed convex bounded subset of $E$. Let $T: C \rightarrow E$ be a mapping which is asymptotically nonexpansive in the intermediate sense. For each $\epsilon>0$ there exsst an integer $K_{\epsilon}>0$ and $\delta_{\epsilon}>0$ such that if $n \geq 2$ is any integer, $j \geq K_{\epsilon}$, $z_{1}, \ldots, z_{n} \in C$ and if $\left\|z_{\imath}-z_{k}\right\|-\left\|T(P T)^{\jmath-1} z_{2}-T(P T)^{\jmath-1} z_{k}\right\| \leq \delta_{\epsilon}$ for $1 \leq i, k \leq n$, then

$$
\left\|T(P T)^{j-1}\left(\sum_{\imath=1}^{n} t_{\imath} z_{\imath}\right)-\sum_{\imath=1}^{n} t_{\imath} T(P T)^{j-1} z_{2}\right\|<\epsilon
$$

for all $t=\left(t_{1}, t_{2}, \ldots t_{n}\right)$ such that $t_{2} \geq 0$ for $i=1,2, \ldots, n$ and $\sum_{\imath=1}^{n} t_{i}=1$.

Proof. The proof follows basically as in the proof of Lemma 2.2 of [22] and is, therefore, omitted.

We now state and prove the following theorems: 
Theorem 3.5. (Demiclosed principle for non-self map) Let $E$ be a uniformly convex Banach space and $K$ a nonempty closed convex subset of $E$. Let $T: K \rightarrow E$ be a mapprng which is asymptotzcally nonexpansive in the intermediate sense. If $\left\{x_{n}\right\}$ is a sequence in $K$ converging weakly to $x^{*}$ and if $\lim _{\jmath \rightarrow \infty}\left(\lim \sup _{n}\left\|x_{n}-T(P T)^{\jmath-1} x_{n}\right\|\right)=0$, then $T x^{*}=x^{*}$.

Proof. This is basically the proof of Lemma 2.5 of Oka [22] (see also, Kaczor et. al [17]). For completeness and because of more general nature of our map, we sketch the details. Clearly, $\left\{x_{n}\right\}$ is bounded. So, there exists $R>0$ such that $\left\{x_{n}\right\} \subset C:=K \cap \bar{B}_{R}(0)$, where $\bar{B}_{R}(0)$ is the closed ball in $E$ with center 0 and radius $R$. Thus, $C$ is a nonempty, closed, bounded, and convex subset of $K$.

\section{Claim:}

$$
T(P T)^{3-1} x^{*} \rightarrow x^{*} \text { as } j \rightarrow \infty \text {. }
$$

For $\epsilon>0$, choose an integer $K_{\frac{\epsilon}{5}}$ and $\delta_{\frac{\varepsilon}{5}}>0$ as in Lemma 3.4. Since $T$ is asymptotically nonexpansive in the intermediate sense and $\lim _{\jmath \rightarrow \infty}\left(\limsup _{n}\left\|x_{n}-T(P T)^{\jmath-1} x_{n}\right\|\right)=0$, there exists an integer $K_{1}(\epsilon)$ such that if $j \geq K_{1}(\epsilon)$, then $\left\|T(P T)^{j-1} u-T(P T)^{j-1} v\right\|-\|u-v\|<\frac{\epsilon}{5}$ for $u, v \in C$ and $\underset{n}{\lim \sup }\left\|x_{n}-T(P T)^{j-1} x_{n}\right\|$ $<\frac{1}{2} \delta_{\frac{\varepsilon}{5}}$. Therefore, there exists $n_{\epsilon, J}$ such that $\left\|x_{n}-T(P T)^{\jmath-1} x_{n}\right\|<\frac{1}{2} \delta_{\frac{\varepsilon}{5}}$ for $n \geq n_{\epsilon, J}$. Put $\epsilon^{\prime}=$ $\min \left\{\frac{1}{2} \delta_{\frac{\sigma}{5}}, \frac{\epsilon}{5}\right\}$. Then we can also take $K_{1}\left(\epsilon^{\prime}\right) \geq 1$. Let $K_{2}(\epsilon)=\max \left\{K_{\frac{\epsilon}{5}}, K_{1}(\epsilon), K_{1}\left(\epsilon^{\prime}\right)\right\}$ and let $k \geq K_{2}(\epsilon)$. Since $\left\{x_{n}\right\}$ converges weakly to $x^{*}$, by Mazur's theorem (see e.g., [34]), we have that for all $n>1$, there exists a convex combination

$$
\begin{aligned}
& y_{n}=\sum_{i=1}^{m(n)} t_{i}^{(n)} x_{2+n}, t_{\imath}^{(n)} \geq 0 \text { and } \sum_{\imath=1}^{m(n)} t_{\imath}^{(n)}=1 \text { such that } \\
& \left\|y_{n}-x^{*}\right\| \rightarrow 0 \text { as } n \rightarrow \infty .
\end{aligned}
$$

Since

$$
\begin{gathered}
\left\|x_{\imath+n}-x_{k+n}\right\|-\left\|T(P T)^{\jmath-1} x_{i+n}-T(P T)^{\jmath-1} x_{k+n}\right\| \\
\leq\left\|x_{\imath+n}-T(P T)^{\jmath-1} x_{\imath+n}\right\|+\left\|x_{k+n}-T(P T)^{\jmath-1} x_{k+n}\right\| \leq \delta_{\frac{5}{5}}
\end{gathered}
$$

for $1 \leq i, k \leq m(n)$, by Lemma 3.4, we have

$$
\left\|T(P T)^{\jmath-1} y_{n}-\sum_{i=1}^{m(n)} t_{i}^{(n)} T(P T)^{\jmath-1} x_{i+n}\right\|<\frac{\epsilon}{5}
$$

since $x_{\imath+n}$ and $x_{k+n}$ are both in $C$. There is also $N_{j, \epsilon} \geq 1$ such that $\left\|y_{n}-x^{*}\right\|<\frac{\epsilon}{5}$ for all $n \geq N_{j, \epsilon}$. Since $x^{*} \in C$,

$$
\begin{aligned}
\left\|T(P T)^{\jmath-1} x^{*}-x^{*}\right\| \leq & \left\|T(P T)^{\jmath-1} x^{*}-T(P T)^{\jmath-1} y_{n}\right\| \\
& +\left\|T(P T)^{\jmath-1} y_{n}-\sum_{i=1}^{m(n)} t_{i}^{(n)} T(P T)^{\jmath-1} x_{\imath+n}\right\| \\
& +\left\|\sum_{i=1}^{m(n)} t_{\imath}^{(n)}\left(T(P T)^{\jmath-1} x_{\imath+n}-x_{i+n}\right)\right\|+\left\|y_{n}-x^{*}\right\|<\epsilon
\end{aligned}
$$

whenever $n \geq N_{\jmath, \epsilon}$ and $j \geq K_{2}(\epsilon)$. Thus $\left\|T(P T)^{\jmath-1} x^{*}-x^{*}\right\|<\epsilon$ for $j \geq K_{2}(\epsilon)$ and so $\| T(P T)^{\jmath-1} x^{*}-$ $x^{*} \| \rightarrow 0$ as $j \rightarrow \infty$. Therefore, (3.5) follows. By the continuity of $T P$, we have that $\lim _{j \rightarrow \infty} T P\left(T(P T)^{j-1} x^{*}\right)=$ $T P x^{*}=T x^{*}=x^{*}$. This completes the proof. 
Theorem 3.6. Let $E$ be a real unformly convex Banach space and $K$ a nonempty closed convex subset of $E$. Let $T: K \rightarrow E$ be a mapping which is asymptotically nonexpansive in the intermediate sense with $c_{n}=\max \left\{0, \sup _{x, y \in K}\left(\left\|T(P T)^{n-1} x-T(P T)^{n-1} y\right\|-\|x-y\|\right)\right\}$ such that $\sum_{n \geq 1} c_{n}<\infty$. Suppose $x^{*} \epsilon$ $F(T):=\{x \in K: T x=x\}$. Let $\left\{\alpha_{n}\right\}_{n \geq 1} \subset(0,1)$ be such that $\epsilon \leq \alpha_{n} \leq 1-\epsilon \forall n \geq 1$ and some $\epsilon>0$. Starting from arbitrary $x_{1} \in K$, define the sequence $\left\{x_{n}\right\}$ by the recursion (3.4). Then $\lim _{n \rightarrow \infty}\left\|x_{n}-x^{*}\right\|$ exists.

Proof. From the recursion (3.4) we have that

$$
\begin{aligned}
\left\|x_{n+1}-x^{*}\right\| & =\left\|P\left(\left(1-\alpha_{n}\right) x_{n}+\alpha_{n} T(P T)^{n-1} x_{n}\right)-P x^{*}\right\| \\
& \leq\left\|\left(1-\alpha_{n}\right) x_{n}+\alpha_{n} T(P T)^{n-1} x_{n}-x^{*}\right\| \\
& \leq\left(1-\alpha_{n}\right)\left\|x_{n}-x^{*}\right\|+\alpha_{n}\left\|T(P T)^{n-1} x_{n}-T(P T)^{n-1} x^{*}\right\| \\
& \leq\left(1-\alpha_{n}\right)\left\|x_{n}-x^{*}\right\|+\alpha_{n}\left(\left\|x_{n}-x^{*}\right\|+c_{n}\right) \\
& \leq\left\|x_{n}-x^{*}\right\|+(1-\epsilon) c_{n}
\end{aligned}
$$

$\forall n \geq 1$. By Lemma 1 of [32], we conclude that $\lim _{n \rightarrow \infty}\left\|x_{n}-x^{*}\right\|$ exists and so is bounded. This completes the proof.

Theorem 3.7. Let $E$ be a real uniformly convex Banach space and $K$ a nonempty closed convex subset of $E$. Let $T: K \rightarrow E$ be a mapping which is asymptotically nonexpansive in the intermediate sense with $c_{n}=\max \left\{0, \sup _{x, y \in K}\left(\left\|T(P T)^{n-1} x-T(P T)^{n-1} y\right\|-\|x-y\|\right)\right\}$ such that $\sum_{n \geq 1} c_{n}<\infty$. Suppose $F(T) \neq \emptyset$. Let $\left\{\alpha_{n}\right\}_{n \geq 1} \subset(0,1)$ be such that $\epsilon \leq \alpha_{n} \leq 1-\epsilon \forall n \geq 1$ and some $\epsilon>0$. From arbitrary $x_{1} \in K$, define the sequence $\left\{x_{n}\right\}$ by the recursion (3.4). Then $\lim _{n \rightarrow \infty}\left\|x_{n}-T x_{n}\right\|=0$.

Proof. Let $x^{*} \in F(T)$ and let $\lim _{n \rightarrow \infty}\left\|x_{n}-x^{*}\right\|=r>0$. Notice $\left\{x_{n}\right\}$ is bounded. So, there exists $R>0$ such that $x_{n} \in B_{R}(0) \forall n \geq 1$.

\section{Claim:}

$$
\lim _{n \rightarrow \infty}\left\|T(P T)^{n-1} x_{n}-x_{n}\right\|=0 .
$$

For this claim, we compute (using Theorem 2 of [37]) as follows:

$$
\begin{aligned}
\left\|x_{n+1}-x^{*}\right\|^{p}= & \left\|P\left(\left(1-\alpha_{n}\right) x_{n}+\alpha_{n} T(P T)^{n-1} x_{n}\right)-P x^{*}\right\|^{p} \\
\leq & \left\|\left(1-\alpha_{n}\right)\left(x_{n}-x^{*}\right)+\alpha_{n}\left(T(P T)^{n-1} x_{n}-x^{*}\right)\right\|^{p} \\
\leq & \left(1-\alpha_{n}\right)\left\|x_{n}-x^{*}\right\|^{p}+\alpha_{n}\left(\left\|x_{n}-x^{*}\right\|+c_{n}\right)^{p} \\
& -W_{p}\left(\alpha_{n}\right) g\left(\left\|T(P T)^{n-1} x_{n}-x_{n}\right\|\right)
\end{aligned}
$$

so that

$$
\begin{aligned}
2 \epsilon^{p+1} g\left(\left\|T(P T)^{n-1} x_{n}-x_{n}\right\|\right) \leq & \left(1-\alpha_{n}\right)\left\|x_{n}-x^{*}\right\|^{p}+\alpha_{n}\left(\left\|x_{n}-x^{*}\right\|+c_{n}\right)^{p} \\
& -\left\|x_{n+1}-x^{*}\right\|^{p} .
\end{aligned}
$$

In particular, for some constant $M>0$,

$$
2 \epsilon^{3} g\left(\left\|T(P T)^{n-1} x_{n}-x_{n}\right\|\right) \leq\left\|x_{n}-x^{*}\right\|^{2}-\left\|x_{n+1}-x^{*}\right\|^{2}+c_{n} M .
$$


Hence

This implies that

$$
\sum_{n \geq 1} g\left(\left\|T(P T)^{n-1} x_{n}-x_{n}\right\|\right)<\infty
$$

$$
\lim _{n \rightarrow \infty} g\left(\left\|T(P T)^{n-1} x_{n}-x_{n}\right\|\right)=0 .
$$

But $g$ is strictly increasing, continuous and $g(0)=0$. Hence

$$
\lim _{n \rightarrow \infty}\left\|T(P T)^{n-1} x_{n}-x_{n}\right\|=0
$$

Furthermore,

$$
\begin{aligned}
\left\|x_{n+1}-x_{n}\right\| & =\left\|P\left(\left(1-\alpha_{n}\right) x_{n}+\alpha_{n} T(P T)^{n-1} x_{n}\right)-P x_{n}\right\| \\
& \leq\left\|\left(1-\alpha_{n}\right) x_{n}+\alpha_{n} T(P T)^{n-1} x_{n}-x_{n}\right\| \\
& =\alpha_{n}\left\|T(P T)^{n-1} x_{n}-x_{n}\right\| \\
& \leq(1-\epsilon)\left\|T(P T)^{n-1} x_{n}-x_{n}\right\| .
\end{aligned}
$$

This implies that $\lim _{n \rightarrow \infty}\left\|x_{n+1}-x_{n}\right\|=0$. Also

$$
\begin{aligned}
\left\|x_{n}-T x_{n}\right\| \leq & \left\|x_{n}-x_{n+1}\right\|+\left\|x_{n+1}-T(P T)^{n} x_{n+1}\right\| \\
& +\left\|T(P T)^{n} x_{n+1}-T(P T)^{n} x_{n}\right\|+\left\|T(P T)^{n} x_{n}-T x_{n}\right\| .
\end{aligned}
$$

Since $T$ is uniformly continuous and $P$ is nonexpansive, we conclude that

$$
\lim _{n \rightarrow \infty}\left\|x_{n}-T x_{n}\right\|=0
$$

This completes the proof.

Theorem 3.8. Let $E$ be a real uniformly convex Banach space and $K$ a nonempty closed convex subset of $E$. Let $T: K \rightarrow E$ be a completely continuous mapping which is asymptotically nonexpansive in the intermediate sense with $c_{n}=\max \left\{0, \sup _{x, y \in K}\left(\left\|T(P T)^{n-1} x-T(P T)^{n-1} y\right\|-\|x-y\|\right)\right\}$ such that $\sum_{n \geq 1} c_{n}<\infty$. Suppose $F(T) \neq \emptyset$. Let $\left\{\alpha_{n}\right\}_{n \geq 1} \subset(0,1)$ be such that $\epsilon \leq \alpha_{n} \leq 1-\epsilon \forall n \geq 1$ for some $\epsilon>0$. From arbitrary $x_{1} \in K$, define the sequence $\left\{x_{n}\right\}$ by the recursion (3.4). Then $\left\{x_{n}\right\}$ converges strongly to some fixed point of $T$.

Proof. Since by Theorem $3.6\left\{x_{n}\right\}$ is bounded and by hypothesis $T$ is completely continuous, there exists a subsequence $\left\{T x_{n_{\jmath}}\right\}$ of $\left\{T x_{n}\right\}$ such that $T x_{n_{j}} \rightarrow y^{*}$ as $j \rightarrow \infty$. Moreover, by Theorem 3.7 we have that $\left\|T x_{n_{3}}-x_{n}\right\| \rightarrow 0$ which implies that $x_{n_{j}} \rightarrow y^{*}$ as $j \rightarrow \infty$. Thus by the continuity of $T, T y^{*}=y^{*}$. Futhermore, since $\lim _{n \rightarrow \infty}\left\|x_{n}-y^{*}\right\|$ exists by Theorem 3.6, the conclusion holds. This completes the proof.

Corollary 3.9. Let $E$ be a real uniformly convex Banach space and $K$ a nonempty closed convex subset of $E$. Let $T: K \rightarrow E$ be completely contrnuous and asymptotically nonexpansive with sequence $\left\{k_{n}\right\}_{n \geq 1} \subset$ $[1, \infty)$ such that $\sum_{n \geq 1}\left(k_{n}-1\right)<\infty$. Suppose $F(T) \neq \emptyset$. Let $\left\{\alpha_{n}\right\}_{n \geq 1} \subset(0,1)$ be such that $\epsilon \leq \alpha_{n} \leq$ $1-\epsilon \forall n \geq 1$ for some $\epsilon>0$. From arbitrary $x_{1} \in K$, define the sequence $\left\{x_{n}\right\}$ by the recursion (3.4). Then $\left\{x_{n}\right\}$ converges strongly to some fixed point of $T$. 
Proof. Since $\left\{x_{n}\right\}$ is bounded, there exists $R>0$ such that $\left\{x_{n}\right\} \subseteq C:=K \cap \overline{B_{r}}(0)$, where $\overline{B_{R}}(0)$ is the closed ball in $E$ with center 0 and radius $R$. As a result, $C$ is a nonempty, closed, bounded, and convex subset of $K$. Define $c_{n}=\max \left\{0, \sup _{x, y \in C}\left(\left\|T(P T)^{n-1} x-T(P T)^{n-1} y\right\|-\|x-y\|\right)\right\}$ and observe that

$$
\sum_{n \geq 1} c_{n} \leq \sum_{n \geq 1}\left(k_{n}-1\right) \operatorname{diam}(C)<\infty
$$

Now Theorem 3.8 guarantees that $\left\{x_{n}\right\}$ converges strongly to some fixed point of $T$. This completes the proof.

A mapping $T: K \rightarrow E$ with $F(T) \neq \emptyset$ is said to satisfy condrtion (A) (see [29]) if there is a nondecreasing function $f:[0, \infty) \rightarrow[0, \infty)$ with $f(0)=0$ and $f(r)>0$ for all $r \in(0, \infty)$ such that for all $x \in K$

$$
\|x-T x\| \geq f(d(x, F(T)))
$$

Theorem 3.10. Let $E$ be a real uniformly convex Banach space and $K$ a nonempty closed convex subset of $E$. Let $T: K \rightarrow E$ be a mapping which is asymptotically nonexpansive in the intermediate sense satisfying condition (A) with $c_{n}=\max \left\{0, \sup _{x, y \in K}\left(\left\|T(P T)^{n-1} x-T(P T)^{n-1} y\right\|-\|x-y\|\right)\right\}$ such that $\sum_{n \geq 1} c_{n}<\infty$. Suppose $F(T) \neq \emptyset$. Let $\left\{\alpha_{n}\right\}_{n \geq 1} \subset(0,1)$ be such that $\epsilon \leq \alpha_{n} \leq 1-\epsilon \forall n \geq 1$ and some $\epsilon>0$. From arbitrary $x_{1} \in K$, define the sequence $\left\{x_{n}\right\}$ by the recursion (3.4). Then $\left\{x_{n}\right\}$ converges strongly to some fixed point of $T$.

Proof. Let $x^{*} \in F(T)$. Then as in Theorem 3.6

$$
\left\|x_{n+1}-x^{*}\right\| \leq\left\|x_{n}-x^{*}\right\|+(1-\epsilon) c_{n}
$$

$\forall n \geq 1$ and $\lim _{n \rightarrow \infty}\left\|x_{n}-x^{*}\right\|$ exists. Since $d\left(x_{n+1}, F(T)\right) \leq d\left(x_{n}, F(T)\right)+(1-\epsilon) c_{n}$, we have that $\lim _{n \rightarrow \infty} d\left(x_{n}, F(T)\right.$ ) exists (again, by Lemma 1 of [32]). Also by Theorem $3.7, \lim _{n \rightarrow \infty}\left\|x_{n}-T x_{n}\right\|=0$. Since $T$ satisfies the condition (A), it follows that

$$
\lim _{n \rightarrow \infty} f\left(d\left(x_{n}, F(T)\right)\right)=0 .
$$

Consequently, we have $\lim _{n \rightarrow \infty} d\left(x_{n}, F(T)\right)=0$. We now prove that $\left\{x_{n}\right\}$ is a Cauchy sequence. For given any $\hat{\epsilon}>0$, there exists a positive integer $N$ such that

$$
d\left(x_{n}, F(T)\right)<\frac{\hat{\epsilon}}{8} \text { and } \sum_{n=N}^{\infty} c_{n}<\frac{\hat{\epsilon}}{4(1-\epsilon)}
$$

$\forall n \geq N$. Now by the definition of infimum, there exists $y^{*} \in F(T)$ such that $\left\|x_{N}-y^{*}\right\|<\frac{\hat{\epsilon}}{4}$. Notice

$$
\begin{aligned}
\left\|x_{n+m}-x_{n}\right\| \leq & \left\|x_{n+m}-y^{*}\right\|+\left\|x_{n}-y^{*}\right\| \leq\left\|x_{N}-y^{*}\right\| \\
& +(1-\epsilon) \sum_{\jmath=N}^{n+m-1} c_{\jmath}+\left\|x_{N}-y^{*}\right\|+(1-\epsilon) \sum_{\jmath=N}^{n-1} c_{\jmath}<\hat{\epsilon}
\end{aligned}
$$

$\forall n \geq N$ and $m \geq 1$. This implies that $\left\{x_{n}\right\}$ is a Cauchy sequence and so there exist $z^{*} \in K$ such that $x_{n} \rightarrow z^{*}$ as $n \rightarrow \infty$. Since $\lim _{n \rightarrow \infty}\left\|x_{n}-T x_{n}\right\|=0$ and $T$ is continuous, we immediately get that $z^{*}=T z^{*}$. This completes the proof.

The following is an immediate consequence of Theorem 3.10. 
Corollary 3.11. Let $E$ be a real uneformly convex Banach space and $K$ a nonempty closed convex subset of $E$. Let $T: K \rightarrow E$ be asymptotically nonexpansive satisfying condition (A) with sequence $\left\{k_{n}\right\}_{n \geq 1} \subset[1, \infty)$ such that $\sum_{n \geq 1}\left(k_{n}-1\right)<\infty$. Suppose $F(T) \neq \emptyset$. Let $\left\{\alpha_{n}\right\}_{n \geq 1} \subset(0,1)$ be such that $\epsilon \leq \alpha_{n} \leq 1-\epsilon \forall n \geq 1$ and some $\epsilon>0$. From arbitrary $x_{1} \in K$, define the sequence $\left\{x_{n}\right\}$ by the recursion (3.4). Then $\left\{x_{n}\right\}$ converges strongly to some fixed point of $T$.

Lemma 3.12. Let $E$ be a real uniformly convex Banach space and $K$ a nonempty closed convex subset of $E$. Let $T: K \rightarrow E$ be a mapping which is asymptotically nonexpansive in the intermediate sense with $c_{n}=\max \left\{0, \sup _{x, y \in K}\left(\left\|T(P T)^{n-1} x-T(P T)^{n-1} y\right\|-\|x-y\|\right)\right\}$ such that $\sum_{n \geq 1} c_{n}<\infty$. Suppose $F(T) \neq \emptyset$. Let $\left\{\alpha_{n}\right\}_{n \geq 1} \subset(0,1)$ be such that $\epsilon \leq \alpha_{n} \leq 1-\epsilon \forall n \geq 1$ and some $\epsilon>0$. From arbatrary $x_{1} \in K$, define the sequence $\left\{x_{n}\right\}$ by the recursion (3.4). Then for all $u, v \in F(T)$, the limit

$$
\lim _{n \rightarrow \infty}\left\|t x_{n}+(1-t) u-v\right\|
$$

exists for all $t \in[0,1]$.

Proof. Let $a_{n}(t)=\left\|t x_{n}+(1-t) u-v\right\|$. Then $\lim _{n \rightarrow \infty} a_{n}(0)=\|u-v\|$, and from Theorem 3.6, $\lim _{n \rightarrow \infty} a_{n}(1)=$ $\lim _{n \rightarrow \infty}\left\|x_{n}-v\right\|$ exists. Without loss of generality, we may assume that $\lim _{n \rightarrow \infty}\left\|x_{n}-u\right\|=r>0$ and $t \in(0,1)$. Define $T_{n}: K \rightarrow K$ by $T_{n} x=P\left(\left(1-\alpha_{n}\right) x+\alpha_{n} T(P T)^{n-1} x\right), \quad x \in K$ and $S_{n, m}:=$ $T_{n+m-1} T_{n+m-2} \ldots T_{n}, m \geq 1$. Then

$$
\left\|S_{n, m} x-S_{n, m} y\right\| \leq\|x-y\|+\sum_{\jmath=n}^{n+m-1} c_{\jmath} .
$$

Observe that $S_{n, m} x_{n}=x_{n+m}$ and $S_{n, m} y=y \forall y \in F(T)$. Set

$b_{n, m}:=\left\|x_{n}-u\right\|\left[S_{n, m}\left(t x_{n}+(1-t) u\right)-t S_{n, m} x_{n}-(1-t) S_{n, m} u\right]$;

$D_{n, m}:=\left[S_{n, m} u+S_{n, m} x_{n}-2 S_{n, m}\left(t x_{n}+(1-t) u\right)\right] \sum_{\jmath=n}^{n+m-1} c_{j}$

$M_{n, m}:=\left[t|| x_{n}-u \|+\sum_{\jmath=n}^{n+m-1} c_{j}\right] \times\left[(1-t)\left\|x_{n}-u\right\|+\sum_{\jmath=n}^{n+m-1} c_{\jmath}\right]$;

$F_{n, m}:=\left[t S_{n, m} u+(1-2 t) S_{n, m}\left(t x_{n}+(1-t) u\right)-(1-t) S_{n, m} x_{n}\right] \times \sum_{\jmath=n}^{n+m-1} c_{\jmath} ;$

$L_{n}:=\left[t|| x_{n}-u \|+L\right] \times\left[(1-t)\left\|x_{n}-u\right\|+L\right]$, where $L=\sum_{\jmath=1}^{\infty} c_{\jmath}$.

It is well known (see, for example, [2]. p. 108) that if $E$ is uniformly convex,

$$
\begin{aligned}
\|t x+(1-t) y\| & \leq 1-2 \min \{t,(1-t)\} \delta_{E}(\|x-y\|) \\
& \leq 1-2 t(1-t) \delta(\|x-y\|)
\end{aligned}
$$

for all $t \in[0,1]$ and for all $x, y \in E$ such that $\|x\| \leq 1,\|y\| \leq 1$. Set

$$
\begin{aligned}
& W_{n, m}:=\frac{S_{n, m} u-S_{n, m}\left(t x_{n}+(1-t) u\right)}{t|| x_{n}-u \|+\sum_{\jmath=n}^{n+m-1} c_{\jmath}} ; \\
& Z_{n, m}:=\frac{S_{n, m}\left(t x_{n}+(1-t) u\right)-S_{n, m} x_{n}}{(1-t)\left\|x_{n}-u\right\|+\sum_{\jmath=n}^{n+m-1} c_{\jmath}} .
\end{aligned}
$$

Then $\left\|W_{n, m}\right\| \leq 1$ and $\left\|Z_{n, m}\right\| \leq 1$ so that it follows from (3.6) that

$$
2 t(1-t) \delta_{E}\left(\left\|W_{n, m}-Z_{n, m}\right\|\right) \leq 1-\left\|t W_{n, m}+(1-t) Z_{n, m}\right\| .
$$


Observe that

$$
\begin{gathered}
\left\|W_{n, m}-Z_{n, m}\right\|=\frac{\left\|b_{n, m}-D_{n, m}\right\|}{M_{n, m}} \text { and } \\
\left\|t W_{n, m}+(1-t) Z_{n, m}\right\|=\frac{\|t(1-t)\| x_{n}-u\left\|\left[S_{n, m} u-S_{n, m} x_{n}\right]+F_{n, m}\right\|}{M_{n, m}} .
\end{gathered}
$$

From (3.7) we then obtain that

$$
\begin{aligned}
2 M_{n, m} \delta_{E}\left(\frac{\left\|b_{n, m}-D_{n, m}\right\|}{M_{n, m}}\right) \leq & \left\|x_{n}-u\right\|^{2} \\
& +\frac{1}{t(1-t)} \sum_{j=n}^{n+m-1} c_{j}\left[\left\|x_{n}-u\right\|+\sum_{j=n}^{n+m-1} c_{j}\right] \\
& -\left\|x_{n}-u\right\| .\left\|x_{n+m}-u\right\|+\frac{\left\|F_{n, m}\right\|}{t(1-t)} .
\end{aligned}
$$

Observe that $L_{n} \geq M_{n, m}$ for all $n, m$. Since $E$ is uniformly convex, then $\frac{\delta_{E}(s)}{s}$ is nondecreasing and hence it follows from (3.8) that

$$
\begin{aligned}
2 L_{n} \delta_{E}\left(\frac{\left\|b_{n, m}-D_{n, m}\right\|}{L_{n}}\right) \leq & \left\|x_{n}-u\right\|^{2} \\
& +\frac{1}{t(1-t)} \sum_{j=n}^{n+m-1} c_{j}\left[\left\|x_{n}-u\right\|+\sum_{j=n}^{n+m-1} c_{j}\right] \\
& -\left\|x_{n}-u\right\| \cdot\left\|x_{n+m}-u\right\|+\frac{\left\|F_{n, m}\right\|}{t(1-t)}
\end{aligned}
$$

Since $\lim _{n \rightarrow \infty}\left\|x_{n}-u\right\|$ exists we have that $\lim _{n \rightarrow \infty}\left\|x_{n}-u\right\|=\lim _{n \rightarrow \infty}\left\|x_{n+m}-u\right\|$. Moreover, since $\delta_{E}(0)=0$, the continuity of $\delta_{E}$ gives from inequality (3.9) that $\liminf \operatorname{insup}_{n}\left(\lim \sup _{n, m}-D_{n, m} \|\right)=0$. Observe that

$$
\begin{aligned}
\underset{m}{\limsup }\left\|b_{n, m}\right\| \leq & \underset{m}{\limsup }\left\|b_{n, m}-D_{n, m}\right\|+\underset{m}{\limsup }\left\|D_{n, m}\right\| \\
& =\underset{m}{\limsup }\left\|b_{n, m}-D_{n, m}\right\|+K_{n} \sum_{j=n}^{\infty} c_{j}
\end{aligned}
$$

for some bounded sequence $\left\{K_{n}\right\}$. Since $\sum_{j=n}^{\infty} c_{j} \rightarrow 0$ as $n \rightarrow \infty$ and $\liminf _{n}\left(\limsup _{m}\left\|b_{n, m}-D_{n, m}\right\|\right)=0$, it follows that $\liminf _{n}\left(\underset{m}{\limsup }\left\|b_{n, m}\right\|\right)=0$. Hence,

$$
\liminf _{n}\left(\limsup _{m}\left\|S_{n, m}\left(t x_{n}+(1-t) u\right)-t S_{n, m} x_{n}-(1-t) S_{n, m} u\right\|\right)=0 .
$$


Clearly,

$$
\begin{aligned}
a_{n+m}(t) \leq & \| t x_{n+m}+(1-t) u-v \\
& +\left(S_{n, m}\left(t x_{n}+(1-t) u\right)-t S_{n, m} x_{n}-(1-t) S_{n, m} u\right) \| \\
& +\left\|-\left[S_{n, m}\left(t x_{n}+(1-t) u\right)-t S_{n, m} x_{n}-(1-t) S_{n, m} u\right]\right\| \\
= & \left\|S_{n, m}\left(t x_{n}+(1-t) u\right)-v\right\|+\| S_{n, m}\left(t x_{n}+(1-t) u\right)-t S_{n, m} x_{n} \\
& -(1-t) S_{n, m} u \| \\
\leq & \left\|t x_{n}+(1-t) u-v\right\|+\sum_{\jmath=n}^{n+m-1} c_{\jmath} \\
& +\left\|S_{n, m}\left(t x_{n}+(1-t) u\right)-t S_{n, m} x_{n}-(1-t) S_{n, m} u\right\| \\
\leq & a_{n}(t)+\sum_{\jmath=n}^{n+m-1} c_{\jmath}+\left\|S_{n, m}\left(t x_{n}+(1-t) u\right)-t S_{n, m} x_{n}-(1-t) S_{n, m} u\right\| .
\end{aligned}
$$

Hence $\lim \sup _{n \rightarrow \infty} a_{n}(t) \leq \liminf _{n \rightarrow \infty} a_{n}(t)$, and the conclusion follows.

Theorem 3.13. Let $E$ be a real uniformly convex Banach space such that its dual $E^{*}$ has the KadecKlee property and $K$ a nonempty closed convex subset of $E$. Let $T: K \rightarrow E$ be a mapping which is asymptotically nonexpansive in the intermediate sense with $c_{n}=\max \left\{0, \sup _{x, y \in K}\left(\| T(P T)^{n-1} x-\right.\right.$ $\left.\left.T(P T)^{n-1} y\|-\| x-y \|\right)\right\}$ such that $\sum_{n \geq 1} c_{n}<\infty$. Suppose $F(T) \neq \emptyset$. Let $\left\{\alpha_{n}\right\}_{n \geq 1} \subset(0,1)$ be such that $\epsilon \leq \alpha_{n} \leq 1-\epsilon \forall n \geq 1$ and some $\epsilon>0$. From arbitrary $x_{1} \in K$, define the sequence $\left\{x_{n}\right\}$ by the recursion (3.4). Then $\left\{x_{n}\right\}$ converges weakly to some fixed point of $T$.

Proof. Theorem 3.6 guarantees that $\left\{x_{n}\right\}$ is bounded. Since $E$ is reflexive, there exists a subsequence $\left\{x_{n_{3}}\right\}$ of $\left\{x_{n}\right\}$ converging weakly to some $x^{*}$. We also have by Theorem 3.7 that $\left\|x_{n_{3}}-T x_{n_{2}}\right\| \rightarrow 0$ as $j \rightarrow \infty$. Since $T$ is uniformly continuous, we can get that

$$
\lim _{m \rightarrow \infty}\left(\limsup _{j}\left\|T(P T)^{m-1} x_{n_{\jmath}}-x_{n},\right\|\right)=0 .
$$

Now Theorem 3.5 guarantees that $T x^{*}=x^{*}$. It remains to show that $\left\{x_{n}\right\}$ converges weakly to $x^{*}$. Suppose $\left\{x_{m_{j}}\right\}$ is another subsequence of $\left\{x_{n}\right\}$ converging weakly to some $y^{*}$. Then $y^{*} \in \bar{K}^{w}=\bar{K}=K$ and so $x^{*}, y^{*} \in \omega_{w}\left(x_{n}\right) \cap F(T)$. By Lemma 3.12, the limit

$$
\lim _{n \rightarrow \infty}\left\|t x_{n}+(1-t) x^{*}-y^{*}\right\|
$$

exists for all $t \in[0,1]$. Now Lemma 2.1 guarantees that $x^{*}=y^{*}$. As a result, $\omega_{w}\left(x_{n}\right)$ is a singleton, and so $\left\{x_{n}\right\}$ converges weakly to a fixed point of $T$.

The following corollaries follow immediately from Theorem 3.13.

Corollary 3.14. Let $E$ be a real unformly convex Banach space such that its dual $E^{*}$ has the Kadec-Klee property and $K$ a nonempty closed convex subset of $E$. Let $T: K \rightarrow E$ be asymptotically nonexpansive with sequence $\left\{k_{n}\right\}_{n \geq 1} \subset[1, \infty)$ such that $\sum_{n \geq 1}\left(k_{n}-1\right)<\infty$. Suppose $F(T) \neq \emptyset$. Let $\left\{\alpha_{n}\right\}_{n \geq 1} \subset(0,1)$ be such that $\epsilon \leq \alpha_{n} \leq 1-\epsilon \forall n \geq 1$ and some $\epsilon>0$. From arbitrary $x_{1} \in K$, define the sequence $\left\{x_{n}\right\}$ by the recursion (3.4). Then $\left\{x_{n}\right\}$ converges weakly to some fixed point of $T$. 
Corollary 3.15. Let E be a real uniformly convex Banach space such that its dual $E^{*}$ has the KadecKlee property and $K$ a nonempty closed convex subset of $E$. Let $T: K \rightarrow E$ be nonexpansive. Suppose $F(T) \neq \emptyset$. Let $\left\{\alpha_{n}\right\}_{n \geq 1} \subset(0,1)$ be such that $\epsilon \leq \alpha_{n} \leq 1-\epsilon \forall n \geq 1$ and some $\epsilon>0$. For arbitrary $x_{1} \in K$, define the sequence $\left\{x_{n}\right\}$ by the recursion (3.4). Then $\left\{x_{n}\right\}$ converges weakly to some $x^{*} \in F(T)$.

Remark 3.16. We remark that if the so-called error terms are added in our recursion formula and are assumed to be bounded, then the results of this paper still hold.

Remark 3.17. It is well known that duals of reflexive Banach spaces with Fréchet differentiable norms have the Kadec-Klee property (see, e.g. [9]). However, it is worth mentioning that there exist uniformly convex Banach spaces which have neither a Fréchet differentiable norm nor satisfy Opial's condition but their duals do have the Kadec-Klee property. To see this, consider $X=\mathbb{R}^{2}$ with the norm given by $|x|=\sqrt{\|x\|_{1}^{2}+\|x\|_{2}^{2}}$ and $Y=L^{p}[0,1]$ with $1<p<\infty$ and $p \neq 2$. Then the Cartesian product $X \times Y$ equipped with the $l^{2}$-norm is uniformly convex, it does not satisfy Opial's condition, and its norm is not Fréchet differentiable. However, its dual does have the Kadec-Klee property. For details, we refer the reader to $[10]$ and $[16]$.

Remark 3.18. Theorem 3.8 extends Theorem 1.5 of Schu [27] and the corresponding results of Kim and Kim [18], Rhoades [26], and Osilike and Aniagbosor [23] to the more general class of non-self maps. Furthermore, no boundedness condition is imposed on $K$ as in [21]. Theorem 3.5 extends Theorem 1 of Oka [22] and Kaczor et al. [17] to the more general class of non-self maps. Under the additional hypothesis that the dual $E^{*}$ of $E$ has the Kadec-Klee property, Theorem 3.13 generalizes Theorem JS to the case of non-self maps in Banach spaces that include $L_{p}$ spaces $(1<p<\infty)$, with Opial's condition and boundedness of $K$ dispensed with. Since duals of reflexive Banach spaces with Fréchet differentiable norms have the Kadec-Klee property, Theorem 3.13 extends Theorem TX to non-self maps which are asymptotically nonexpansive in the intermediate sense with boundedness of $K$ dispensed with. Theorem 3.13 generalizes and improves the results of [23] to the more general class of non-self maps, and without the assumption that $(I-T)$ is demiclosed and that $E$ satisfies Opial condition. The results of this paper also extend and improve the corresponding results of [7].

Acknowledgements This work was done while the authors (NS) and (HZ) were visiting the Abdus Salam International Centre for Theoretical Physics, Trieste, Italy. They would like to thank the Centre for hospitality and financial support. This work was done within the framework of the Associateship Scheme of the Abdus Salam ICTP. 


\section{REFERENCES}

[1] S. C. Bose, Weak convergence to a fixed point of an asymptotically nonexpansive map, Proc. Amer. Math. Soc., 68 (1978), 305-308.

[2] R. E. Bruck, A simple proof of the mean ergodic theorem for nonlinear contractions in Banach spaces, Isreal J. Math., 32 (1979), 107-116.

[3] R. E. Bruck, T. Kuczumow, and S. Reich, Convergence of iterates of asymptotically nonexpansive mappings in Banach spaces with the uniform Opial property, Colloq. Math., 65 (1993), 169-179.

[4] S. S. Chang, Y. J. Cho and H. Zhou, Demi-closed principle and weak convergence problems for asymptotically nonexpansive mappings, J. Korean Math. Soc., 38 (2001), No. 6, 1245-1260.

[5] C. E. Chidume, Nonexpansive mappings, generalizations and iterative algorithms, Nonlinear Anal. and Appl., (to appear).

[6] C. E. Chidume, On the approximation of fixed points of nonexpansive mappings, Houston J. Math., 7(1981), 345-355.

[7] C. E. Chidume, E. U. Ofoedu and H. Zegeye, Strong and weak convergence theorems for asymptotically nonexpansive mappings, J. Math. Anal. Appl., 280 (2003), 364-374.

[8] C. E. Chidume, N. Shahzad and H. Zegeye, Strong convergence theorems for nonexpansive mappings in arbitrary Banach spaces, Nonlinear Anal., submitted.

[9] J. Diestel, Geometry of Banach Spaces-Selected Topics, Lecture Notes in Mathematics, Vol. 485, Springer Verlag, New York, 1975.

[10] J. G. Falset, W. Kaczor, T. Kuczumow, and S. Reich, Weak convergence theorems for asymptotically nonexpansive mappings and semigroups, Nonlinear Anal., 43 (2001), 377-401.

[11] T. Figiel, On the moduli of convexity and smoothness, Studia Math., 56 (1976), 121-155.

[12] K. Goebel and W. A. Kirk, A fixed point theorem for asymptotically nonexpansive mappings, Proc. Amer. Math. Soc., 35 (1972), 171-174.

[13] J. Górnicki, Nonlinear ergodic theorems for asymptotically nonexpansive mappings in Banach spaces satisfying Opial's condition, J. Math. Anal. Appl., 161 (1991), 440-446.

[14] S. Ishikawa, Fixed points and iteration of a nonexpansive mapping in a Banach space, Proc. Amer. Math. Soc., 59 (1976), 65-71.

[15] S. Ishikawa, Fixed points by a new iteration method, Proc. Amer. Math. Soc., 44 (1974), 147-150.

[16] W. Kaczor, Weak convergence of almost orbits of asymptotically nonexpansive commutative semigroups, J. Math. Anal. Appl., 272 (2002), 565-574.

[17] W. Kaczor, T. Kuczumow, and S. Reich, A mean ergodic theorem for mappings which are asymptotically nonexpansive in the intermediate sense, Nonlinear Anal., 47 (2001), 2731-2742.

[18] G. E. Kim and T. H. Kim, Mann and Ishikawa iterations with errors for non-Lipschitian mappings in Banach spaces, Comput. Math. Appl., 42 (2001), 1565-1570.

[19] W. A. Kirk, Fixed point theorems for non-Lipschitian mappings of asymptotically nonexpansive type, Israel J. Math., 17 (1974), 339-346.

[20] J. Lindenstrauss and L. Tzafriri, Classical Banach Spaces II, Springer Verlag, New York, 1979.

[21] W. R. Mann, Mean value methods in iteration, Proc. Amer. Math. Soc., 4 (1953), 506-510.

[22] H. Oka, An ergodic theorem for asymptotically nonexpansive mappings in the intermediate sense, Proc. Amer. Math. Soc., 125 (1997), 1693-1703.

[23] M. O. Osilike and S. C. Aniagbosor, Weak and strong convergence theorems for fixed points of asymptotically nonexpansive mappings, Math. Comput. Modelling, 32 (2000), 1181-1191.

[24] G. B. Passty, Construction of fixed points for asymptotically nonexpansive mappings, Proc. Amer. Math. Soc., 84 (1982), 212-216.

[25] S. Reich, Weak convergence theorems for nonexpansive mappings in Banach spaces, J. Math. Anal. Appl., 67 (1979), 274-276.

[26] B. E. Rhoades, Fixed point iterations for certain nonlinear mappings, J. Math. Anal. Appl., 183 (1994), 118-120.

[27] J. Schu, Iterative construction of fixed points of asymptotically nonexpansive mappings, J. Math. Anal. Appl., 158 (1991), 407-413.

[28] J. Schu, Weak and strong convergence of fixed points of asymptotically nonexpansive mappings, Bull. Austral. Math. Soc., 43 (1991), 153-159.

[29] H. F. Senter and W. G. Dotson, Approximating fixed points of nonexpansive mappings, Proc. Amer. Math. Soc., 44 (1974), 375-380. 
[30] K. K. Tan and H. K. Xu, A nonlinear ergodic theorem for asymptotically nonexpansive mappings, Bull. Austral. Math. Soc., 45 (1992), 25-36.

[31] K. K. Tan and H. K. Xu, The nonlinear ergodic theorem for asymptotically nonexpansive mapping in Banach spaces, Proc. Amer. Math. Soc., 114 (1992), 399-404.

[32] K. K. Tan and H. K. Xu, Approximating fixed points of nonexpansive mappings by the Ishikawa iteration process, J. Math. Anal. Appl., 178 (1993), 301-308.

[33] K. K. Tan and H. K. Xu, Fixed point iteration processes for asymptotically nonexpansive mappings, Proc. Amer. Math. Soc., 122 (1994), 733-739.

[34] P. Wojtaszczyk, Banach spaces for analysts, Cambridge University Press, Cambridge, 1991.

[35] Z. B. Xu and G. F. Roach, Characteristic inequalities of uniformly convex and uniformly smooth Banach spaces, J. Math. Anal. Appl., 157 (1991), 189-210.

[36] $\mathrm{H}$. K. Xu, Existence and convergence for fixed points of mappings of asymptotically nonexpansive type, Nonlinear Anal., 16 (1991), 1139-1146.

[37] H. K. Xu, Inequalities in Banach spaces with applications, Nonlnear Anal., 16 (1991), 1127-1138. 